\title{
Optimal control of switched autonomous linear systems
}

\author{
Alessandro Giua (*), Carla Seatzu (*), Cornelis Van Der Mee (**) \\ (*) Dip. di Ing. Elettrica ed Elettronica, $(* *)$ Dip. di Matematica — Università di Cagliari, Italy \\ Phone: +39-070-675-5892 - Fax: +39-070-675-5900 - Email: \{giua,seatzu\}@ diee.unica.it; cornelis@ krein.unica.it
}

\begin{abstract}
The paper deals with the optimal control of switched piecewise linear autonomous systems, where the objective is that of minimizing a quadratic performance index over an infinite time horizon. We assume that the switching sequence and the corresponding jump matrices sequence is known, while the unknown switching times are the optimization parameters. The optimal control for this class of systems, assuming a switching sequence of finite length, takes the form of a homogeneous state feedback, i.e., it is possible to identify a homogeneous region of the state space such that an optimal switch should occur if and only if the present state belongs to this region; we show how such a region can be computed with a numerical procedure. As the number of allowed switches goes to infinity, we study the stability of the system and discuss some preliminary results related to the convergence of the state feedback law.
\end{abstract}

\section{Introduction}

Switched systems are a particular class of hybrid systems [1] consisting of a certain number of subsystems (that may also be infinite) and a switching law that indicates the active subsystem at each time instant. Examples of switched systems may be found in many application fields, such as chemical processes, transportation systems, electrical circuit systems, and so on.

The problem of determining optimal control laws for this class of hybrid systems has been widely investigated in the last years and many results can be found in the control and computer science literature $[2,5,7,10]$. Many of these works propose control procedures that are based on the discretization of state space into grids and use search methods to find optimal open-loop solutions. We also mention the contribution given by Riedinger et al. in [7, 8] where very general sufficient conditions for optimal control problems of switched systems are given in terms of hamiltonian function.

In this paper we restrict our attention to the case of switched systems whose subsystems are linear and autonomous. We assume that we have a pre-assigned switching sequence between autonomous linear dynamics of the type $\dot{\boldsymbol{x}}(t)=\boldsymbol{A}_{j} \boldsymbol{x}(t)$, where the sequence $\boldsymbol{A}_{j}, j=1,2, \cdots$ is known but the switching times $\tau_{j}$ are unknown. We also generalize this framework by assuming that whenever at time $\tau_{j}$ a switch from $\boldsymbol{A}_{j}$ to $\boldsymbol{A}_{j+1}$ occurs, the state should jump from $\boldsymbol{x}\left(\tau_{j}^{-}\right)$to $\boldsymbol{x}\left(\tau_{j}^{+}\right)=\boldsymbol{M}_{j} \boldsymbol{x}\left(\tau_{j}^{-}\right)$. We make the following assumptions:

a) Each matrix $\boldsymbol{A}_{j}$ is stable, thus an optimal choice of the $\tau_{j}$ 's will ensure the stability of the switched system under some hypothesis on the structure of the allowed jumps (we prove this).

b) Each system is autonomous, i.e., we do not need to compute a continuous control. The only control input for this system is the controlled switch/jump. Also switch and jump are coupled, in the sense that the $j$-th jump and the $j$-th switch are triggered by the same event and occur at the same time.

c) We assume that no cost is associated to a switch.
The control problem consists in determining the optimal switching times $\tau_{j}, j=1,2, \cdots$, so as to minimize a quadratic performance index of the form:

$$
F\left(\tau_{1}, \tau_{2}, \cdots\right)=\int_{0}^{\infty} \boldsymbol{x}^{T}(t) \boldsymbol{Q} \boldsymbol{x}(t) d t
$$

where $Q$ is a positive definite matrix.

When only a finite number $n$ of switches may take place, the results we presented in [4] show that the optimal control law turns out to be a "homogeneous feedback", in the sense that for all $j \leq n$ : (a) it is possible to identify a region $\mathcal{C}_{j, n}$ of the state space such that the $j$-th switch should occur if and only if we are within this region; (b) this region is homogeneous, i.e., if $\boldsymbol{x} \in \mathcal{C}_{j, n}$ then $\lambda \boldsymbol{x} \in \mathcal{C}_{j, n}$, for all real numbers $\lambda$. In [4], we also considered the case in which a cost is associated to each switching and studied how the regions are correspondingly modified. The original features of our approach can be summarized as follows. Firstly, our derivation is based on the analytical derivation of the cost functional rather than the hamiltonian. Secondly, we are able to show that in this particular case the optimal control is a state feedback (and not an open-loop control). Thirdly, we are able to compute with a simple numerical procedure not only necessary but also sufficient conditions for optimality.

In this paper, we consider the case in which the number of allowed switches $n$ goes to infinity. In this case, stability issues become important because the stability of each subsystem is neither a sufficient nor a necessary condition for the stability of the overall system; this topic has been studied in the literature $[3,6,9]$. We show that the switched system controlled with the proposed procedure is stable in the sense of Lyapunov because of the optimality of the control law if we assume that the number of subsystems $\mathcal{A}=\left\{\boldsymbol{A}_{j} \mid j \geq 1\right\}$ is finite and the jump matrices $\boldsymbol{M}_{j}$ satisfy a not very restrictive condition.

However, a different type of instability, zenoness, may appear if we allow consecutive switching times $\tau_{j}=\tau_{j+1}=$ $\cdots=\tau_{j+r}$ to take the same value. To rule out this case, we propose in this paper a modified procedure that finds the optimal control law under the constraint that $\tau_{j}-\tau_{j-1} \geq \delta>0$, $j=1,2, \cdots$ (spacing condition). Even for the modified procedure we prove the stability of the controlled systems under possibly infinite switchings.

Finally, we also study the convergence properties of the switching regions for an infinite number of switches. The preliminary results presented in the paper do not give a constructive algorithm to determine the regions.

One limitation of the present approach is the fact that the switching sequence is pre-assigned. In effect, preliminary results that are not discussed here, show that our approach can easily be generalized to consider a (possibly infinite) set of legal sequences provided that they can all be generated by a finite state automaton over the alphabet $\mathcal{A}$. We observe, however, that there exist significant problems of practical relevance where the present framework (pre-assigned sequence) may be successfully applied. Consider, as an example, an active filtering problem where by connecting or disconnecting a capacitor 
one aims to reduce the distortion of an output signal. Such a problem can be framed as a pre-assigned sequence of switches $\boldsymbol{A} \rightarrow \tilde{\boldsymbol{A}} \rightarrow \boldsymbol{A} \rightarrow \cdots$, where $\dot{\boldsymbol{x}}(t)=\boldsymbol{A x}(t)$ is the dynamics of the system with the capacitor connected and $\dot{\boldsymbol{x}}(t)=\tilde{\boldsymbol{A}} \boldsymbol{x}(t)$ is the dynamics of the system with the capacitor disconnected.

It may be possible to extend the results we present here to the cases - considered in the literature already mentioned where the subsystem dynamics are not all stable but there exists a stabilizing switching sequence; this is a topic for future work.

\section{The System with Switching Conditions}

\subsection{System Dynamics}

Given the switching times $0=\tau_{0} \leq \tau_{1} \leq \cdots \leq \tau_{n} \leq$ $\tau_{n+1}=\infty$, the $N \times N$ stable matrices $\boldsymbol{A}_{1}, \cdots, \boldsymbol{A}_{n+1} \in \overline{\mathcal{A}}$ and the switching matrices $\boldsymbol{M}_{1}, \cdots, \boldsymbol{M}_{n}$, consider the linear system whose dynamics are given by

$$
\left\{\begin{array}{l}
\dot{\boldsymbol{x}}(t)=\boldsymbol{A}_{j} \boldsymbol{x}(t), \quad \tau_{j-1}<t<\tau_{j}, \\
\boldsymbol{x}\left(\tau_{k}^{+}\right)=\boldsymbol{M}_{k} \boldsymbol{M}_{k-1} \cdots \boldsymbol{M}_{j} \boldsymbol{x}\left(\tau_{j}^{-}\right) \\
\quad \text { for } \tau_{j-1}<\tau_{j}=\cdots=\tau_{k}<\tau_{k+1}, \\
\boldsymbol{x}(0)=\boldsymbol{x}_{0}
\end{array}\right.
$$
by

Then we define the evolution matrices $\boldsymbol{U}(t, \tau)(t>\tau \geq 0)$

$$
\boldsymbol{x}\left(t^{-}\right)=\boldsymbol{U}(t, \tau) \boldsymbol{x}\left(\tau^{+}\right) .
$$

Then, obviously, for $\tau_{j-1}<\tau_{j}=\cdots=\tau_{k}<\tau_{k+1}$ :

$$
\boldsymbol{U}\left(\tau_{k}^{+}, \tau\right)=\boldsymbol{M}_{k} \boldsymbol{M}_{k-1} \cdots \boldsymbol{M}_{j} \boldsymbol{U}\left(\tau_{j}, \tau\right)
$$

and

$$
\boldsymbol{U}\left(t, \tau_{j}^{-}\right)=\boldsymbol{U}\left(t, \tau_{k}\right) \boldsymbol{M}_{k} \boldsymbol{M}_{k-1} \cdots \boldsymbol{M}_{j}
$$

Let us define $\delta_{j}=\tau_{j}-\tau_{j-1}(j=1, \cdots, n)$. One easily verifies that

$$
\begin{aligned}
\boldsymbol{U}(t, \tau)= & e^{\boldsymbol{A}_{k+1}\left(t-\tau_{k}\right)} \boldsymbol{M}_{k} e^{\boldsymbol{A}_{k} \delta_{k}} \ldots \\
& \cdots \boldsymbol{M}_{j+1} e^{\boldsymbol{A}_{j+1} \delta_{j+1}} \boldsymbol{M}_{j} e^{\boldsymbol{A}_{j}\left(\tau_{j}-\tau\right)}
\end{aligned}
$$

whenever $\tau_{j-1} \leq \tau<\tau_{j} \leq \tau_{j+1} \leq \cdots \leq \tau_{k}<t \leq \tau_{k+1}$.

\subsection{Optimization problem}

Given a positive definite $N \times N$ matrix $\boldsymbol{Q}$, we define the cost functional

$$
F\left(\tau_{1}, \cdots, \tau_{n}\right)=\int_{0}^{\infty} \boldsymbol{x}^{T}(t) \boldsymbol{Q x}(t) d t
$$

Clearly, using the convention $\boldsymbol{U}(+\infty, \tau)=0$ and since

$$
\int_{\tau_{j-1}}^{\tau_{j}} e^{\boldsymbol{A}_{j}^{T}\left(t-\tau_{j-1}\right)} \boldsymbol{Q} e^{\boldsymbol{A}_{j}\left(t-\tau_{j-1}\right)} d t=\left[e^{\boldsymbol{A}_{j}^{T} t} \boldsymbol{Z}_{j} e^{\boldsymbol{A}_{j} t}\right]_{\delta_{j}}^{0}
$$

where $Z_{j}$ are the unique solutions of the Lyapunov equations

$$
\boldsymbol{A}_{j}^{T} \boldsymbol{Z}_{j}+\boldsymbol{Z}_{j} \boldsymbol{A}_{j}=-\boldsymbol{Q}
$$

we get

$$
\begin{aligned}
& F\left(\tau_{1}, \cdots, \tau_{n}\right)=\boldsymbol{x}_{0}^{T} \boldsymbol{Z}_{1} \boldsymbol{x}_{0} \\
& \quad+\sum_{j=1}^{n} \boldsymbol{x}^{T}\left(\tau_{j}^{-}\right)\left[\boldsymbol{M}_{j}^{T} \boldsymbol{Z}_{j+1} \boldsymbol{M}_{j}-\boldsymbol{Z}_{j}\right] \boldsymbol{x}\left(\tau_{j}^{-}\right) .
\end{aligned}
$$

Using the relations

$$
\frac{\partial \boldsymbol{x}\left(\tau_{k}^{-}\right)}{\partial \tau_{j}}= \begin{cases}0, & k<j \\ \boldsymbol{A}_{j} \boldsymbol{x}\left(\tau_{j}^{-}\right), & k=j \\ \boldsymbol{U}\left(\tau_{k}, \tau_{j}\right)\left[-\boldsymbol{A}_{j+1} \boldsymbol{M}_{j}+\boldsymbol{M}_{j} \boldsymbol{A}_{j}\right] \boldsymbol{x}\left(\tau_{j}^{-}\right), & k>j\end{cases}
$$

we obtain

$$
\frac{\partial}{\partial \tau_{j}} F\left(\tau_{1}, \cdots, \tau_{n}\right)=\boldsymbol{x}^{T}\left(\tau_{j}^{-}\right) \boldsymbol{G}_{j, n} \boldsymbol{x}\left(\tau_{j}^{-}\right),
$$

where

$$
\begin{aligned}
\boldsymbol{G}_{j, n} & =\boldsymbol{A}_{j}^{T}\left[\boldsymbol{M}_{j}^{T} \boldsymbol{Z}_{j+1} \boldsymbol{M}_{j}-\boldsymbol{Z}_{j}\right] \\
& +\left[\boldsymbol{M}_{j}^{T} \boldsymbol{Z}_{j+1} \boldsymbol{M}_{j}-\boldsymbol{Z}_{j}\right] \boldsymbol{A}_{j} \\
& +\left[-\boldsymbol{M}_{j}^{T} \boldsymbol{A}_{j+1}^{T}+\boldsymbol{A}_{j}^{T} \boldsymbol{M}_{j}^{T}\right] \boldsymbol{H}_{j, n} \\
& +\boldsymbol{H}_{j, n}\left[-\boldsymbol{A}_{j+1} \boldsymbol{M}_{j}+\boldsymbol{M}_{j} \boldsymbol{A}_{j}\right]
\end{aligned}
$$

and

$$
\begin{aligned}
& \boldsymbol{H}_{j, n}=\sum_{i=j+1}^{n} \boldsymbol{U}\left(\tau_{i}, \tau_{j}\right)^{T}\left[\boldsymbol{M}_{i}^{T} \boldsymbol{Z}_{i+1} \boldsymbol{M}_{i}-\boldsymbol{Z}_{i}\right] \boldsymbol{U}\left(\tau_{i}, \tau_{j}\right) \\
& =\sum_{i=j+1}^{n} e^{\boldsymbol{A}_{j+1}^{T} \delta_{j+1}} \boldsymbol{M}_{j+1}^{T} \cdots \boldsymbol{M}_{i-1}^{T} e^{\boldsymbol{A}_{i}^{T} \delta_{i}} \times \\
& \times\left[\boldsymbol{M}_{i}^{T} \boldsymbol{Z}_{i+1} \boldsymbol{M}_{i}-\boldsymbol{Z}_{i}\right] e^{\boldsymbol{A}_{i} \delta_{i}} \boldsymbol{M}_{i-1} \cdots \boldsymbol{M}_{j+1} e^{\boldsymbol{A}_{j+1} \delta_{j+1}}
\end{aligned}
$$

for $j=1, \cdots, n-1$ and $\boldsymbol{H}_{n, n}=\mathbf{0}$.

\section{State-feedback control law}

From eq. (6) it follows that an optimal $j-$ th switch may only occur in the region of the state space defined by

$$
\tilde{\mathcal{C}}_{j, n}=\left\{\boldsymbol{x} \in \mathbb{R}^{N} \mid \boldsymbol{x}^{T} \boldsymbol{G}_{j, n} \boldsymbol{x} \geq 0\right\}
$$

that includes the points where a delay in the switching time leads to an increase in the cost functional. Thus we assume that the following law is used to enforce the $j$-th switch:

- if the $(j-1)$-th switch leads to a state $\boldsymbol{x}_{j-1} \notin \tilde{\mathcal{C}}_{j, n}$ the state will evolve until a point $\boldsymbol{x}_{j}$ on the boundary of $\tilde{\mathcal{C}}_{j, n}$ is reached and the $j$-th switch will occur there;

- if the $(j-1)$-th switch leads to a state $\boldsymbol{x}_{j-1}$ internal to $\tilde{\mathcal{C}}_{j, n}$, the $j$-th switch will occur immediately, i.e., $\boldsymbol{x}_{j}=$ $\boldsymbol{x}_{j-1}$.

Now, we first observe that while for the $n$-th (i.e., the last) switch, $\boldsymbol{G}_{n, n}$ is a matrix of constants, from eq. (7) it follows that for all $j<n$ the value of $\boldsymbol{G}_{j, n}\left(\delta_{j+1}, \cdots, \delta_{n}\right)$ depends on the future intervals between switching times. Therefore, we define for $j=1, \cdots, n$ the function: $\Delta_{j}: \mathbb{R}^{N} \rightarrow[0, \infty]$ where

$$
\Delta_{j}(\boldsymbol{x})=\min \left\{\delta \in[0, \infty] \mid e^{\boldsymbol{A}_{j} \delta} \boldsymbol{x} \in \tilde{\mathcal{C}}_{j, n}\right\},
$$

is the length of the shortest time interval necessary to reach starting from $\boldsymbol{x}$ and with dynamics given by $\boldsymbol{A}_{j}-$ a point on $\tilde{\mathcal{C}}_{j, n}$. Two limit cases are possible: $\Delta_{j}(\boldsymbol{x})=0$ if $\boldsymbol{x} \in \tilde{\mathcal{C}}_{j, n}$, while $\Delta_{j}(\boldsymbol{x})=\infty$ if $\tilde{\mathcal{C}}_{j, n}$ is not reached in finite time. 
The corresponding switching law can thus be described in terms of the following switching regions $(j<n)$ :

$$
\begin{aligned}
& \tilde{\mathcal{C}}_{n, n}=\left\{\boldsymbol{x} \in \mathbb{R}^{N} \mid \boldsymbol{x}^{T} \boldsymbol{G}_{n, n} \boldsymbol{x} \geq 0\right\}, \\
& \tilde{\mathcal{C}}_{j, n}=\left\{\boldsymbol{x} \in \mathbb{R}^{N} \mid \exists \delta_{j+1} \cdots, \delta_{n},\right. \text { such that : } \\
& \quad \delta_{j+1}=\Delta_{j+1}(\boldsymbol{x}) ; \\
& \quad \delta_{j+2}=\Delta_{j+2}\left(\boldsymbol{M}_{j+1} e^{\boldsymbol{A}_{j+1} \delta_{j+1}} \boldsymbol{x}\right) ; \\
& \quad \cdots \\
& \quad \delta_{n}=\Delta_{n}\left(\boldsymbol{M}_{n-1} e^{\left.\boldsymbol{A}_{n-1} \delta_{n-1} \cdots \boldsymbol{M}_{j+1} e^{\boldsymbol{A}_{j+1} \delta_{j+1}} \boldsymbol{x}\right) ;}\right. \\
& \left.\quad \boldsymbol{x}^{T} \boldsymbol{G}_{j, n}\left(\delta_{j+1}, \cdots, \delta_{n}\right) \boldsymbol{x} \geq 0\right\} .
\end{aligned}
$$

An important result follows immediately.

Proposition 3.1 The regions $\tilde{\mathcal{C}}_{j, n}(j=1, \cdots, n)$ are homogeneous spaces, i.e., $\boldsymbol{x} \in \tilde{\mathcal{C}}_{j, n} \Longrightarrow(\forall \lambda \in \mathbb{R}) \lambda \boldsymbol{x} \in \tilde{\mathcal{C}}_{j, n}$.

Proof: We prove this by induction on $j$. Clearly $\tilde{\mathcal{C}}_{n, n}$ is homogeneous, because $\boldsymbol{G}_{n, n}$ is a constant matrix (base step). Assume now that all $\tilde{\mathcal{C}}_{i, n}(i=j+1, \cdots, n)$ are homogeneous. This clearly implies, given the characterization of (9), that the function $\Delta_{i}(i=j+1, \cdots, n)$ are such that $\Delta_{i}(\boldsymbol{x})=\Delta_{i}(\lambda \boldsymbol{x})$ for all $0 \neq \lambda \in \mathbb{R}$. This in turn implies that $\tilde{\mathcal{C}}_{j, n}$ is homogeneous (induction step). We conclude that $\tilde{\mathcal{C}}_{j, n}$ is a homogeneous space for all $j=1, \cdots, n$.

Note that this characterization provides an algorithmic way to construct the regions. We observe that it is sufficient to determine which points on the unitary semi-sphere belong to a region to completely determine the region (because it is a homogeneous space). Thus, we choose a suitable discretization step and for each point $\boldsymbol{x}$ on the unitary semi-sphere we determine if it belongs to $\tilde{\mathcal{C}}_{n, n}, \tilde{\mathcal{C}}_{n-1, n}$, etc., also computing step by step the corresponding values of $\Delta_{n}(\boldsymbol{x}), \Delta_{n-1}(\boldsymbol{x})$, etc. This procedure may be burdensome but can be applied off-line. The on-line controller, on the contrary, will simply need to test if the actual state vector belongs to the next switching region.

At this point, we observe that this is a necessary condition for optimality, but in general it is not sufficient. To overcome this restriction, we show how a new set of linear regions can be computed, such that if every switch occurs as soon as they are reached, then optimality is guaranteed. In the rest of the paper they are denoted as $\mathcal{C}_{j, n}, j=1, \cdots, n$.

Once again, these switching regions have to be computed starting from the last one. More precisely, let us first define the residual cost from the $k$-th to $n$-th switch, given the initial state $\boldsymbol{x}$, as:

$$
\begin{aligned}
& F_{k}\left(\boldsymbol{x}, \delta_{k}, \delta_{k+1}, \cdots, \delta_{n}\right)=\boldsymbol{x}^{T} \boldsymbol{Z}_{k} \boldsymbol{x} \\
& \quad+\sum_{j=k}^{n} \boldsymbol{x}_{j}^{T}\left[\boldsymbol{M}_{j}^{T} \boldsymbol{Z}_{j+1} \boldsymbol{M}_{j}-\boldsymbol{Z}_{j}\right] \boldsymbol{x}_{j}
\end{aligned}
$$

with $\boldsymbol{x}_{j}=e^{\boldsymbol{A}_{j} \delta_{j}} \boldsymbol{x}_{j-1}$ and $\boldsymbol{x}_{k-1}=\boldsymbol{x}$. We also define the corresponding $k$-th optimal switching interval as:

$$
\delta_{k}^{*}(\boldsymbol{x})=\arg \min _{\delta_{k} \in \mathbb{R}_{0}^{+}} F_{k}\left(\boldsymbol{x}, \delta_{k}, \delta_{k+1}^{*}\left(\boldsymbol{x}_{k}\right), \cdots, \delta_{n}^{*}\left(\boldsymbol{x}_{n-1}\right)\right)
$$

where $\boldsymbol{x}_{j}=e^{\boldsymbol{A}_{j} \delta_{j}^{*}\left(\boldsymbol{x}_{j-1}\right)} \boldsymbol{x}_{j-1}$. Finally we can write that

$$
\mathcal{C}_{j, n}=\left\{\boldsymbol{x} \mid \delta_{j}^{*}(\boldsymbol{x})=0\right\} \quad(j=1, \cdots, n)
$$

Note that, as in the previous case, the computation of these regions is performed off-line, starting from $\mathcal{C}_{n, n}$ and going backwards.

To conclude, we formally prove an important result.
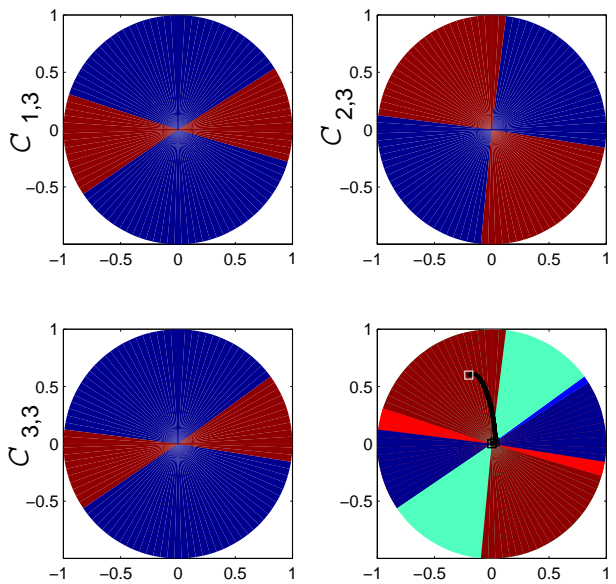

Figure 1: The switching regions $\mathcal{C}_{j, 3}, j=1,2,3$ for the system in the example and the system evolution for $\boldsymbol{x}_{0}=[-0.2,0.6]$.

Proposition 3.2 The regions $\mathcal{C}_{j, n}(j=1, \cdots, n)$ are homogeneous spaces, i.e., $\boldsymbol{x} \in \mathcal{C}_{j, n} \Longrightarrow(\forall \lambda \in \mathbb{R}) \lambda \boldsymbol{x} \in \tilde{\mathcal{C}}_{j, n}$.

Proof: It is enough to show that for $\lambda \in \mathbb{R}$, all $k \in \mathbb{N}$ and all $\boldsymbol{x} \in \mathbb{R}^{N}: \delta_{k}^{*}(\lambda \boldsymbol{x})=\delta_{k}^{*}(\boldsymbol{x})$. Following the same reasoning of Proposition 3.1, this follows immediately from the definition of $\delta^{*}$ and the expression of $F_{k}$ given in (11) that is quadratic in $\boldsymbol{x}$.

\subsection{Example}

Let us consider a second order system whose dynamics may only switch between two matrices $\boldsymbol{A}^{(1)}$ and $\boldsymbol{A}^{(2)}$. We also assume that only three switchings are possible $(n=3)$ and the initial system dynamics is $\boldsymbol{A}^{(1)}$. Thus, the sequence of switching is $\boldsymbol{A}^{(1)} \rightarrow \boldsymbol{A}^{(2)} \rightarrow \boldsymbol{A}^{(1)} \rightarrow \boldsymbol{A}^{(2)}$, where

$$
\boldsymbol{A}^{(1)}=\left[\begin{array}{cc}
-1 & 1 \\
-18 & -5
\end{array}\right], \quad \boldsymbol{A}^{(2)}=\left[\begin{array}{cc}
1 & -5 \\
1 & -3
\end{array}\right] \text {. }
$$

Note that $\boldsymbol{A}^{(1)}$ and $\boldsymbol{A}^{(2)}$ are stable non-commuting matrices, i.e., $\boldsymbol{A}^{(1)} \boldsymbol{A}^{(2)} \neq \boldsymbol{A}^{(2)} \boldsymbol{A}^{(1)}$. We also assume that all $\boldsymbol{M}_{j}$ are equal to the identity matrix.

The switching regions $\mathcal{C}_{j, n}, j=1,2,3$, are shown in figure 1 where the following color notation has been used: the red (lighter) region represents the set of states where the system switches to the next dynamics, while the blue (darker) region represents the set of states where the system still evolves with the same dynamics.

In the bottom right of figure 1 we have shown the system evolution in the case of $\boldsymbol{x}_{0}=[-0.2,0.6]$. The switching times are $\tau_{1}=0.61, \tau_{2}=1.34$ and $\tau_{3}=1.49$, and the optimal cost is $F\left(\tau_{1}, \tau_{2}, \tau_{3}\right)=0.19$.

\section{A stability result}

In this section we consider the case in which $n=\infty$, i.e., the number of allowed switches goes to infinity. As well known from the literature, if a system switches an infinite number of times among a finite number of stable dynamics, then the resulting system is not guaranteed to be stable. Stability in general depends on the switching sequence and on the switching time instants. 
We consider an infinite switching sequence of autonomous linear and stable dynamics as given by equation (1) with $n=\infty$ and make two additional assumptions:

A1: each $\boldsymbol{A}_{j}$ may only take values in a finite set $\mathcal{A}=$ $\left\{\boldsymbol{A}^{(1)}, \boldsymbol{A}^{(2)}, \cdots, \boldsymbol{A}^{(p)}\right\}$;

A2: the sequence of jump matrices $M$ 's is such that

$$
\inf _{j \geq 1} \underline{\sigma}\left(M_{1}\right) \underline{\sigma}\left(M_{2}\right) \cdots \underline{\sigma}\left(M_{j}\right)>0
$$

where $\underline{\sigma}(\boldsymbol{M})$ denotes the smallest singular value of matrix $M$.

Note that the second assumption is always verified when the jump matrices are all equal to the identity matrix.

To prove that the controlled system is stable if the switching times are chosen so as to minimize the cost functional $F$ given by equation (3), we introduce a Lyapunov-like function

$$
V(\boldsymbol{x}, j)=\min \left\{F_{j}\left(\boldsymbol{x}, \delta_{j}, \delta_{j+1}, \cdots\right) \mid \delta_{k} \in \mathbb{R}_{0}^{+}, k \geq j\right\},
$$

where $F_{j}$ is defined in (11); the value of this function coincides with the optimal residual cost associated with a given evolution that starts in $\boldsymbol{x}$ and evolves along the residual sequence $\boldsymbol{A}_{j}, \boldsymbol{A}_{j+1}, \cdots$.

Before providing a lower and upper bound for $V(\boldsymbol{x}, j)$, we present an elementary lemma that will be used in the following derivation.

Lemma 4.1 Given a linear and stationary dynamical system $\dot{\boldsymbol{x}}(t)=\boldsymbol{A} \boldsymbol{x}(t)$, with initial condition $\boldsymbol{x}(0)=\boldsymbol{x}_{0}$, for all $t \geq 0$ holds

$$
\|\boldsymbol{x}(t)\|=\left\|e^{\boldsymbol{A} t} \boldsymbol{x}_{0}\right\| \geq e^{-\sigma t}\left\|\boldsymbol{x}_{0}\right\|,
$$

where $\|\cdot\|$ denotes the Euclidean norm and $\sigma=\|A\|$ is the largest singular value of matrix $\boldsymbol{A}$.

Proof: First observe that (here $\langle\cdot, \cdot\rangle$ denotes the scalar product)

$$
\begin{aligned}
& 2\|\boldsymbol{x}(t)\| \frac{d}{d t}\|\boldsymbol{x}(t)\|=\frac{d}{d t}\langle\boldsymbol{x}(t), \boldsymbol{x}(t)\rangle \geq-2\|\dot{\boldsymbol{x}}(t)\|\|\boldsymbol{x}(t)\| \\
& =-2\|\boldsymbol{A} \boldsymbol{x}(t)\|\|\boldsymbol{x}(t)\| \geq-2\|\boldsymbol{A}\|\|\boldsymbol{x}(t)\|^{2}=-2 \sigma\|\boldsymbol{x}(t)\|^{2},
\end{aligned}
$$

so that $\frac{d}{d t}\|\boldsymbol{x}(t)\| \geq-\sigma\|\boldsymbol{x}(t)\|$. Hence $e^{\sigma t}\|\boldsymbol{x}(t)\|$ is nondecreasing, i.e., $e^{\sigma t}\|\boldsymbol{x}(t)\| \geq\left\|\boldsymbol{x}_{0}\right\|$.

Proposition 4.2 Let $\hat{\sigma}=\max \left\{\left\|\boldsymbol{A}^{(i)}\right\| \mid i=1 \cdots, p\right\}$, be the maximum amongst all largest singular values of all matrices in $\mathcal{A}$, let

$$
\boldsymbol{v}=\arg \min _{\|\boldsymbol{x}\|=1} \boldsymbol{x}^{T} \boldsymbol{Q} \boldsymbol{x}
$$

be the vector on the unitary sphere with minimal quadratic cost, and, said $\boldsymbol{M}_{0}=\boldsymbol{I}$, let $M=$ $\inf _{h, k \geq 0}\left\{\underline{\sigma}\left(M_{h}\right) \underline{\sigma}\left(M_{h+1}\right) \cdots \underline{\sigma}\left(M_{h+k}\right)\right\}$. Note that assumption $A 2$ implies that $M>0$.

Let $\boldsymbol{Z}^{(i)}$ be the solution of $\boldsymbol{A}^{(i)^{T}} \boldsymbol{Z}^{(i)}+\boldsymbol{Z}^{(i)} \boldsymbol{A}^{(i)}=-\boldsymbol{Q}$.

(1) We define

$$
V_{\min }(\boldsymbol{x})=M^{2}\left(\boldsymbol{v}^{T} \boldsymbol{Q} \boldsymbol{v}\right)\left(\int_{0}^{\infty} e^{-2 \hat{\sigma} t} d t\right)\|\boldsymbol{x}\|^{2},
$$

and claim that $V(\boldsymbol{x}, j) \geq V_{\min }(\boldsymbol{x})$ for all $j \geq 1$.

(2) We define

$$
V_{\max }(\boldsymbol{x})=\max _{i=1, \cdots, p}\left\{\boldsymbol{x}^{T} \boldsymbol{Z}^{(i)} \boldsymbol{x} \mid \boldsymbol{A}^{(i)} \in \mathcal{A}\right\},
$$

and claim that $V(\boldsymbol{x}, j) \leq V_{\max }(\boldsymbol{x})$ for all $j \geq 1$.
Proof: The first claim follows from the fact that $V_{\min }$ is associated to the cost of an evolution along the direction of $\boldsymbol{v}$ in which the quadratic form $\boldsymbol{x}^{T} Q \boldsymbol{x}$ is minimized and that is traversed with the fastest decaying rate (associated to $\hat{\sigma}$ ) amongst all those possible with the allowed dynamics and the greatest norm reduction allowed by consecutive jumps. To show this formally, let $\boldsymbol{x}_{o p}(\cdot)$ be an optimal trajectory starting at time $\tau_{j-1}$ from $\boldsymbol{x}$ and location $j$; it holds

$$
\begin{aligned}
& V(\boldsymbol{x}, j)=\int_{\tau_{j-1}}^{\infty} \boldsymbol{x}_{o p}^{T}(t) \boldsymbol{Q} \boldsymbol{x}_{o p}(t) d t \geq \int_{\tau_{j-1}}^{\infty}\left\|\boldsymbol{x}_{o p}(t)\right\|^{2} \boldsymbol{v}^{T} \boldsymbol{Q} \boldsymbol{v} d t \\
& =\left(\boldsymbol{v}^{T} \boldsymbol{Q} \boldsymbol{v}\right) \sum_{i=j}^{\infty} \int_{\tau_{i-1}}^{\tau_{i}} \| e^{\boldsymbol{A}_{i}\left(t-\tau_{i-1}\right)} \boldsymbol{M}_{i-1} \\
& \cdot e^{\boldsymbol{A}_{i-1}\left(\tau_{i-1}-\tau_{i-2}\right)} \boldsymbol{M}_{i-2} \cdots \boldsymbol{M}_{j} e^{\boldsymbol{A}_{j}\left(\tau_{j}-\tau_{j-1}\right)} \boldsymbol{x} \|^{2} d t \\
& \geq\left(\boldsymbol{v}^{T} \boldsymbol{Q} \boldsymbol{v}\right)\left(\sum_{i=j}^{\infty} \underline{\sigma}^{2}\left(\boldsymbol{M}_{i-1}\right) \cdots \underline{\sigma}^{2}\left(\boldsymbol{M}_{j}\right)\right. \\
& \left.\cdot \int_{c}^{\tau_{i}} e^{-2 \hat{\sigma}\left(t-\tau_{i-1}\right)} \cdots e^{-2 \hat{\sigma}\left(\tau_{j}-\tau_{j-1}\right)} d t\right)\|\boldsymbol{x}\|^{2} \\
& \geq M^{2}\left(\boldsymbol{v}^{T} \boldsymbol{Q} \boldsymbol{v}\right)\left(\sum_{i=j}^{\infty} \int_{\tau_{i-1}}^{\tau_{i}} e^{-2 \hat{\sigma}\left(t-\tau_{j-1}\right)} d t\right)\|\boldsymbol{x}\|^{2} \\
& =M^{2}\left(\boldsymbol{v}^{T} \boldsymbol{Q} \boldsymbol{v}\right)\left(\int_{\tau_{j-1}}^{\infty} e^{-2 \hat{\sigma}\left(t-\tau_{j-1}\right)} d t\right)\|\boldsymbol{x}\|^{2} \\
& =M^{2}\left(\boldsymbol{v}^{T} \boldsymbol{Q} \boldsymbol{v}\right)\left(\int_{0}^{\infty} e^{-2 \hat{\sigma} t} d t\right)\|\boldsymbol{x}\|^{2}=V_{\min }(\boldsymbol{x}) .
\end{aligned}
$$

The second claim follows from the fact that starting from $\boldsymbol{x}$ with the dynamics given by $\boldsymbol{A}_{j}$ and without switching, one may obtain a cost $\boldsymbol{x}^{T} \boldsymbol{Z}_{j} \boldsymbol{x} \leq V_{\max }(\boldsymbol{x})$. Clearly, the optimal cost $V(\boldsymbol{x}, j)$ can only be smaller.

Now, we state an obvious monotonicity result.

Property 4.3 Monotonicity Property. Let $\tau, \tau^{\prime} \in \mathbb{R}^{+}$be two generic time instants. If $\tau<\tau^{\prime}$ and the switched system evolves along an optimal trajectory $\boldsymbol{x}_{o p}(\cdot), j_{o p}(\cdot)$, then

$$
V\left(\boldsymbol{x}_{o p}(\tau), j_{o p}(\tau)\right) \geq V\left(\boldsymbol{x}_{o p}\left(\tau^{\prime}\right), j_{o p}\left(\tau^{\prime}\right)\right),
$$

i.e., the optimal remaining cost does not increase along any optimal trajectory.

Proof: Trivially follows from the fact that the cost is the integral of a positive definite function.

From the above monotonicity property, it immediately derives the following stability result.

Proposition 4.4 The switched system considered in this section and optimally controlled is stable because given an arbitrary $\rho>0$, there exists $\gamma>0$ such that $\left\|\boldsymbol{x}\left(\tau^{\prime}\right)\right\| \leq \rho$ for all $\tau^{\prime}>\tau_{j}$ if $\left\|\boldsymbol{x}\left(\tau_{j}\right)\right\| \leq \gamma$.

Proof: Let $C$ be the maximal value for which the curve $V_{\min }(\boldsymbol{x})=C$ is all contained within the closed ball of radius $\rho$. Then, choose $\gamma$ as the minimal value of $\|\boldsymbol{x}\|$ for $\boldsymbol{x}$ belonging to the curve $V_{\max }(\boldsymbol{x})=C$. We prove that any optimal evolution that starts in $\boldsymbol{x}_{o p}\left(\tau_{j}\right)=\boldsymbol{x}_{j}$ within the closed ball of radius $\gamma$ (this initial state is such that $V_{\max }\left(\boldsymbol{x}_{j}\right) \leq C$ ) remains in the closed ball of radius $\rho$. Assume, by contradiction that for $\tau^{\prime} \geq \tau_{j}$, and $j\left(\tau^{\prime}\right)=j^{\prime}$ we have $\boldsymbol{x}_{o p}\left(\tau^{\prime}\right)=\boldsymbol{x}^{\prime}$, with $\left\|\boldsymbol{x}^{\prime}\right\|>\rho$. Then by the first claim of the lemma, $V\left(\boldsymbol{x}^{\prime}, j^{\prime}\right) \geq V_{\min }\left(\boldsymbol{x}^{\prime}\right)>$ $C$, hence $V\left(\boldsymbol{x}^{\prime}, j^{\prime}\right)>V_{\max }\left(\boldsymbol{x}_{j}\right) \geq V\left(\boldsymbol{x}_{j}, j\right)$ where the last inequality follows from the second claim of the lemma, thus contradicting the monotonicity property that states that the cost must decrease along any trajectory. 
A final comment. Due to the discontinuity caused by the jumps, $\boldsymbol{x}(\tau)$ may not be defined if $\tau$ is a switching instant. The results of Property 4.3 and Proposition 4.4 hold if we assume at all points of discontinuity $\boldsymbol{x}(\tau)=\frac{1}{2}\left(\boldsymbol{x}\left(\tau^{-}\right)+\boldsymbol{x}\left(\tau^{+}\right)\right)$.

\section{Non-zeno optimal control}

In the previous section, no limit was posed on the number of switches that may occur simultaneously or within a given finite time interval. This may result in a new type of instability (divergence) that is often called zenoness in the hybrid systems literature. One way to rule out the possibility of a zeno execution is that of assuming that at most one switch may occur within a time interval of length $\delta>0$, i.e., we pose the following spacing condition

$$
\delta_{j}=\tau_{j+1}-\tau_{j} \geq \delta>0, \quad j=1,2,3, \cdots .
$$

Note that this condition is not an abstract assumption, deriving from the necessity of avoiding zeno models, but is in many cases a realistic assumption on the behavior of physical systems, where the dynamics associated to the actuator that produces the switch may not be neglectable with respect to the system dynamics.

When a finite number of switches are allowed we can still compute the optimal switching regions with a slight modification of the procedure described in Section 3. We need to define:

$$
\bar{\delta}_{k}^{*}(\boldsymbol{x})=\arg \min _{\delta_{k} \geq \delta} F_{k}\left(\boldsymbol{x}, \delta_{k}, \bar{\delta}_{k+1}^{*}\left(\boldsymbol{x}_{k}\right), \cdots, \bar{\delta}_{n}^{*}\left(\boldsymbol{x}_{n-1}\right)\right)
$$

where $\boldsymbol{x}_{j}=e^{\boldsymbol{A}_{j} \bar{\delta}_{j}^{*}\left(\boldsymbol{x}_{j-1}\right)} \boldsymbol{x}_{j-1}$ and $F_{k}$ is still given by (11). Finally we can write

$$
\overline{\mathcal{C}}_{j, n}=\left\{\boldsymbol{x} \mid \bar{\delta}_{n}^{*}(\boldsymbol{x})=0\right\} .
$$

The same procedure previously described may be used to compute these regions.

We can also prove the stability of the corresponding control law (that we call non-zeno) for $n=\infty$ with a similar argument to that used in the previous section. We consider an infinite switching sequence of autonomous linear and stable dynamics as given by equation (1) with $n=\infty$ and make the additional assumptions:

A1: each $\boldsymbol{A}_{j}$ may only take values in a finite set $\mathcal{A}=$

$$
\left\{\boldsymbol{A}^{(1)}, \boldsymbol{A}^{(2)}, \cdots, \boldsymbol{A}^{(p)}\right\} \text {. }
$$

Note that in this case the assumption A2 previously defined is not necessary to prove stability.

Finally, we define

$$
\bar{V}(\boldsymbol{x}, j)=\min \left\{F_{j}\left(\boldsymbol{x}, \delta_{j}, \delta_{j+1}, \cdots\right) \mid \delta_{k} \geq \delta, k \geq j\right\},
$$

that coincides with the optimal residual cost for the non-zeno control law associated with a given evolution that starts in $\boldsymbol{x}$ and evolves along the residual sequence $\boldsymbol{A}_{j}, \boldsymbol{A}_{j+1}, \cdots$. Note that in the definition of $\bar{V}(\boldsymbol{x}, j)$ we assume that no switch is possible for $t \in[0, \delta]$, because this would imply $\delta_{j}<\delta$.

It is still immediate to prove this cost has still as upper bound $V_{\max }(\boldsymbol{x})$ defined in Proposition 4.2, while a lower bound is given by $\bar{V}_{\min }=\left(\boldsymbol{v}^{T} \boldsymbol{Q} \boldsymbol{v}\right)\left(\int_{0}^{\delta} e^{-2 \hat{\sigma} t} d t\right)\|\boldsymbol{x}\|^{2}$. Repeating the argument of Proposition 4.4 it is immediate to prove stability for the system controlled with a non-zeno optimal law.

\subsection{Some convergence results}

We have proved the stability of the switched system in the case of an infinite number of switchings when it is optimally controlled. We study for this case the convergence properties of the proposed control procedure. The preliminary results presented here are limited to the convergence of the regions $\tilde{\mathcal{C}}$ (not the regions $\mathcal{C}$ or $\overline{\mathcal{C}}$ ) and do not give a constructive algorithm to determine them.

The stability of the optimally controlled systems implies that for certain $C>0$ and $\varepsilon>0$

$$
\|\boldsymbol{U}(t, \tau)\| \leq C e^{-\varepsilon(t-\tau)}, \quad t>\tau \geq 0 .
$$

If we assume that the constant

$$
Z=\sup _{j=1,2,3, \cdots} \max \left(\left\|\boldsymbol{M}_{j}\right\|,\left\|\boldsymbol{Z}_{j}\right\|\right)<+\infty,
$$

we obtain the absolute convergence of the infinite series

$$
\begin{aligned}
& \boldsymbol{H}_{j}=\sum_{i=j+1}^{\infty} \boldsymbol{U}\left(\tau_{i}, \tau_{j}\right)^{T}\left[\boldsymbol{M}_{i}^{T} \boldsymbol{Z}_{i+1} \boldsymbol{M}_{i}-\boldsymbol{Z}_{i}\right] \boldsymbol{U}\left(\tau_{i}, \tau_{j}\right) \\
& =\sum_{i=j+1}^{\infty} e^{\boldsymbol{A}_{j+1}^{T} \delta_{j+1}} \boldsymbol{M}_{j+1}^{T} \cdots \boldsymbol{M}_{i-1}^{T} e^{\boldsymbol{A}_{i}^{T} \delta_{i}} \times \\
& \times\left[\boldsymbol{M}_{i}^{T} \boldsymbol{Z}_{i+1} \boldsymbol{M}_{i}-\boldsymbol{Z}_{i}\right] e^{\boldsymbol{A}_{i} \delta_{i}} \boldsymbol{M}_{i-1} \cdots \boldsymbol{M}_{j+1} e^{\boldsymbol{A}_{j+1} \delta_{j+1}}
\end{aligned}
$$

whenever the spacing condition (13) is satisfied for a fixed $\delta$. Indeed, a straightforward estimate yields

$$
\begin{aligned}
& \sum_{i=j+1}^{\infty} \| \boldsymbol{U}\left(\tau_{i}, \tau_{j}\right)^{T} {\left[\boldsymbol{M}_{i}^{T} \boldsymbol{Z}_{i+1} \boldsymbol{M}_{i}-\boldsymbol{Z}_{i}\right] \boldsymbol{U}\left(\tau_{i}, \tau_{j}\right) \| } \\
& \leq\left(Z^{3}+Z\right) C^{2} \sum_{i=j+1}^{\infty} e^{-2 \varepsilon\left(\tau_{i}-\tau_{j}\right)}
\end{aligned}
$$

which is convergent as a result of the ratio test and condition (13).

Note that the condition given by (16) is always verified when the sets $\mathcal{A}=\left\{A_{j} \mid j \geq 1\right\}$ and $\mathcal{M}=\left\{M_{j} \mid j \geq 1\right\}$ are finite.

In this case (and under condition (13)) we can study the situation of infinitely many switching times. We then have

$$
\frac{\partial}{\partial \tau_{j}} F\left(\tau_{1}, \tau_{2}, \cdots\right)=\boldsymbol{x}^{T}\left(\tau_{j}^{-}\right) \boldsymbol{G}_{j} \boldsymbol{x}\left(\tau_{j}^{-}\right),
$$

where

$$
\begin{aligned}
& \boldsymbol{G}_{j}=\boldsymbol{A}_{j}^{T}\left[\boldsymbol{M}_{j}^{T} \boldsymbol{Z}_{j+1} \boldsymbol{M}_{j}-\boldsymbol{Z}_{j}\right]+\left[\boldsymbol{M}_{j}^{T} \boldsymbol{Z}_{j+1} \boldsymbol{M}_{j}-\boldsymbol{Z}_{j}\right] \boldsymbol{A}_{j} \\
& \quad+\left[-\boldsymbol{M}_{j}^{T} \boldsymbol{A}_{j+1}^{T}+\boldsymbol{A}_{j}^{T} \boldsymbol{M}_{j}^{T}\right] \boldsymbol{H}_{j}+\boldsymbol{H}_{j}\left[-\boldsymbol{A}_{j+1} \boldsymbol{M}_{j}+\boldsymbol{M}_{j} \boldsymbol{A}_{j}\right]
\end{aligned}
$$

\section{Additional stability properties}

In the previous section we have discussed the stability of the optimally controlled switched system. In this section we present more general results related to the stability of arbitrary switching policies.

The following result, that is also discussed in [9] but presented here for completeness, gives sufficient conditions under which (15) and (16) are satisfied for hybrid systems with continuous switching (i.e., where $\boldsymbol{M}_{j} \equiv \boldsymbol{I}$, being $\boldsymbol{I}$ the identity matrix.). 
Proposition 6.1 Suppose the set $\mathcal{A}=\left\{\boldsymbol{A}_{j} \mid j \geq 1\right\}$ is finite and consists of p pairwise commuting stable matrices $\boldsymbol{A}^{(1)}, \boldsymbol{A}^{(2)}, \cdots, \boldsymbol{A}^{(p)}$, and let the switching matrices $\boldsymbol{M}_{j}$ all be equal to the identity matrix. Then conditions (15) and (16) are satisfied. In particular, the series (17) are absolutely convergent whenever the spacing condition (13) is satisfied.

Proof: Clearly, under the assumptions of this proposition we have

$$
\boldsymbol{U}(t, \tau)=\prod_{i=1}^{p} e^{\boldsymbol{A}^{(i)}\left[\delta_{i}(t)-\delta_{i}(\tau)\right]},
$$

where $\delta_{i}(t)$ is the time within $[0, t]$ spent in the system with state space matrix $\boldsymbol{A}^{(i)}$. Here note that $\sum_{i=1}^{p} \delta_{i}(t)=t$. Since $\boldsymbol{A}^{(1)}, \cdots, \boldsymbol{A}^{(p)}$ have only eigenvalues with real part $<-\varepsilon$ (for some positive $\varepsilon$ ), there exist constants $C_{1}, \cdots, C_{p}$ such that $\left\|e^{A^{(i)} t}\right\| \leq C_{i} e^{-\varepsilon t}(i=1, \cdots, p)$. We then find condition (15), where $C=C_{1} \cdots C_{p}$. Condition (16) is obvious.

Now, let us consider the hybrid system

$$
\begin{cases}\dot{\boldsymbol{x}}(t)=\boldsymbol{A}_{j} \boldsymbol{x}(t), & \tau_{j-1}<t<\tau_{j} \\ \boldsymbol{x}\left(\tau_{j}^{+}\right)=\boldsymbol{M}_{j} \boldsymbol{x}\left(\tau_{j}^{-}\right), & \boldsymbol{x}\left(0^{+}\right)=\boldsymbol{x}_{0}\end{cases}
$$

with state space matrices $\boldsymbol{A}_{j}$, switching matrices $\boldsymbol{M}_{j}$ and switching times $\tau_{j}$. Then by a similarity we mean a sequence of nonsingular matrices $\boldsymbol{S}_{j}$ converting (19) into the new hybrid system

$$
\begin{cases}\dot{\dot{x}}(t)=\check{\boldsymbol{A}}_{j} \check{x}(t), & \tau_{j-1}<t<\tau_{j} \\ \check{\boldsymbol{x}}\left(\tau_{j}^{+}\right)=\check{\boldsymbol{M}}_{j} \check{\boldsymbol{x}}\left(\tau_{j}^{-}\right), & \check{\boldsymbol{x}}\left(0^{+}\right)=\check{\boldsymbol{x}}_{0}\end{cases}
$$

where

$$
\begin{cases}\check{\boldsymbol{x}}(t)=\boldsymbol{S}_{j} \boldsymbol{x}(t) \text { for } \tau_{j-1}<t<\tau_{j}, & \check{\boldsymbol{x}}_{0}=\boldsymbol{S}_{1} \boldsymbol{x}_{0} \\ \boldsymbol{A}_{j}=\boldsymbol{S}_{j} \boldsymbol{A}_{j} \boldsymbol{S}_{j}^{-1}, & \check{\boldsymbol{M}}_{j}=\boldsymbol{S}_{j+1} \boldsymbol{M}_{j} \boldsymbol{S}_{j}^{-1} .\end{cases}
$$

Let us now convert the hybrid system (19) with nonsingular switching matrices $\boldsymbol{M}_{j}$ into the hybrid system (20) such that the new switching matrices $\check{M}_{j}$ are all equal to the identity matrix. This requires choosing $\boldsymbol{S}_{j}$ such that $\boldsymbol{S}_{j+1} \boldsymbol{M}_{j} \boldsymbol{S}_{j}^{-1}=$ $\boldsymbol{I}$. Given a nonsingular matrix $\boldsymbol{S}_{1}$, one must then choose

$$
\boldsymbol{S}_{j+1}=\boldsymbol{S}_{1}\left[\boldsymbol{M}_{j} \boldsymbol{M}_{j-1} \cdots \boldsymbol{M}_{2} \boldsymbol{M}_{1}\right]^{-1}
$$

One easily verifies that

$$
\begin{aligned}
\boldsymbol{U}(t, \tau)= & \boldsymbol{S}_{n+1}^{-1} e^{\check{\boldsymbol{A}}_{n+1}\left(t-\tau_{n}\right)} \check{\boldsymbol{M}}_{n} \\
& \cdot e^{\check{\boldsymbol{A}}_{n}\left(\tau_{n}-\tau_{n-1}\right)} \check{\boldsymbol{M}}_{n-1} \cdots \check{\boldsymbol{M}}_{j} e^{\check{\boldsymbol{A}}_{j}\left(\tau_{j}-t\right)} \boldsymbol{S}_{j} \\
= & \boldsymbol{S}_{n+1}^{-1} e^{\check{\boldsymbol{A}}_{n+1}\left(t-\tau_{n}\right)} e^{\check{\boldsymbol{A}}_{n}\left(\tau_{n}-\tau_{n-1}\right) \cdots e^{\boldsymbol{\boldsymbol { A }}_{j}\left(\tau_{j}-t\right)} \boldsymbol{S}_{j}} \\
= & \boldsymbol{S}_{n+1}^{-1} \check{\boldsymbol{U}}(t, \tau) \boldsymbol{S}_{j},
\end{aligned}
$$

whenever $\tau_{j-1} \leq \tau<\tau_{j}<\tau_{j+1}<\cdots<\tau_{n}<t \leq \tau_{n+1}$. Here $\check{\boldsymbol{U}}(t, \tau)$ is the evolution system pertaining to (20).

Theorem 6.2 Let the set of matrices $M_{j} M_{j-1} \cdots M_{2} M_{1}$ $(j=1,2,3, \cdots)$ and the set of their inverses $\left[\boldsymbol{M}_{j} \boldsymbol{M}_{j-1} \cdots \boldsymbol{M}_{2} \boldsymbol{M}_{1}\right]^{-1}(j=1,2,3, \cdots)$ both be bounded. Then the hybrid system (19) satisfies conditions (15) and (16) if and only if the hybrid system (20) does.
Proof: In view of (21) and (22), the sequence of similarity matrices $\boldsymbol{S}_{j}$ and the sequence of their inverses $\boldsymbol{S}_{j}^{-1}$ are both bounded. The statement of this theorem is then an immediate consequence of (22).

Remark 6.3 Theorem 6.2 allows us to reduce the problem of stating conditions for the stability of an arbitrary hybrid system with nonsingular switching matrices $\boldsymbol{M}_{j}(j=1,2, \cdots)$ to that for a hybrid system where $\boldsymbol{M}_{j} \equiv \boldsymbol{I}(j=1,2, \cdots)$. In particular, if the transformation from the system (19) to the system (20) leads to a hybrid system (20) with finitely many different pairwise commuting matrices $\tilde{\boldsymbol{A}}^{(1)}, \cdots, \tilde{\boldsymbol{A}}^{(p)}$, system (19) satisfies conditions (15) and (16).

\section{Conclusions and future work}

In this paper we dealt with the optimal control of switched piece-wise linear autonomous systems, where the objective is that of minimizing a quadratic performance index over an infinite time horizon. We assumed that the switching sequence is known and the unknowns to be determined are the optimal switching times. The proposed control procedure is based on the computation of homogeneous regions of the state space such that an optimal switch should occur if and only if the present state belongs to this region.

The results obtained so far are interesting, and we hope that they could be extended to a more general setting. In particular we also plan to study in the future the case in which the switching sequence is not pre-assigned and the case in which the subsystems dynamics are neither autonomous nor stable.

\section{References}

[1] P.J. Antsaklis (ed.), "Special Issue on Hybrid Systems: Theory and Applications," Proc. of the IEEE, Vol. 88, No. 7, Jul 2000.

[2] M.S. Branicky, S.K. Mitter, "Algorithms for optimal hybrid control", Proc. 34th IEEE Conf. on Decision and Control, pp. 2661-6, Dec 1995.

[3] M.S. Branicky, "Multiple Lyapunov Functions and Other Analysis Tools for Switched and Hybrid Systems", IEEE Trans. on Automatic Control, Vol. 43, pp. 475-82, 1998.

[4] A. Giua, C. Seatzu, C. Van Der Mee, "Optimal Control of Autonomous Linear Systems Switched with a Pre-Assigned Finite Sequence", Proc. 2001 IEEE Int. Symp. on Intelligent Control (Mexico City, Mexico), September 2001.

[5] S. Hedlund, A. Rantzer, "Optimal Control of Hybrid Systems", Proc. 38th IEEE Conf. on Decision and Control (Phoenix, Arizona), pp. 3972-7, Dec 1999.

[6] B. Hu, X. Xu, A.N. Michel, P.J. Antsaklis, "Stability Analysis for a Class of Nonlinear Switched Systems", Proc. 38th IEEE Conf. on Decision and Control (Phoenix, Arizona), pp. 4374-9, Dec 1999.

[7] P. Riedinger, C. Zanne, F. Kratz "Time Optimal Control of Hybrid Systems", Proc. 1999 American Control Conference (San Diego, CA, USA), Jun 1999.

[8] P. Riedinger, F. Kratz, C. Iung, C. Zanne, "Linear Quadratic Optimization for Hybrid Systems", Proc. 38th IEEE Conf. on Decision and Control (Phoenix, Arizona), pp. 3059-64, Dec 1999.

[9] X. Xu, P.J. Antsaklis, "Design of stabilizing control laws for second-order switched systems", Proc. 14th IFAC World Congress (Beijing, China), Jul 1999.

[10] L.Y. Wang, A. Beydoun, J. Cook, J. Sun, I. Kolmanovsky, “Optimal Hybrid Control with Applications to Automative Powertrain Systems", LNCIS 22, Springer-Verlag, 1997. 